\title{
PRODUTOS FLORESTAIS NÃO MADEIREIROS E VALOR POTENCIAL DE EXPLORAÇÃO SUSTENTÁVEL DA FLORESTA ATLÂNTICA NO SUL DE SANTA CATARINA
}

\author{
NON-TIMBER FOREST PRODUCTS AND SUSTAINABLE EXPLORATION POTENTIAL IN A \\ TROPICAL RAIN FOREST IN SANTA CATARINA STATE, BRAZIL
}

\author{
Guilherme Alves Elias ${ }^{1}$ Robson dos Santos ${ }^{2}$
}

\begin{abstract}
RESUMO
Este estudo indicou os produtos florestais não madeireiros (PFNM) associados a espécies arbóreas dos remanescentes de Floresta Ombrófila Densa estudados no Sul de Santa Catarina. As espécies analisadas foram hierarquizadas com base na análise de valor potencial de exploração sustentável (VPES) dos PFNM, integrando-se às seguintes informações obtidas a partir de revisão bibliográfica: densidade, parte usada, capacidade de regeneração natural e crescimento, conhecimento sobre biologia reprodutiva e dinâmica populacional, processamento requerido e nível de injúria ou toxicidade. Os resultados obtidos evidenciam ser possível a conservação e desenvolvimento regional, a partir de alternativas sustentáveis de uso dos recursos florestais. Das 79 espécies observadas, 38 atingiram VPES igual ou superior a 10, sendo consideradas de elevado potencial de exploração sustentável de PFNM.
\end{abstract}

Palavras-chave: Floresta Ombrófila Densa; biodiversidade; VPES; recursos naturais.

\begin{abstract}
This study indicated the non-timber forest products (NTFPs) associated with tree species of the remnants of Tropical Rain Forest studied in southern Santa Catarina. The species examined were ranked according to their potential value for sustainable exploitation (PVSE) of NTFP, applying the following information obtained from literature review: density, parts used, capacity for natural regeneration and growth, knowledge about reproductive biology and population dynamic, required processing and level of injury or toxicity. The results show possible conservation and regional development, from alternative sustainable use of forest resources. Of the 79 species observed, 38 reached PVSE equal or higher than 10, being considered of high potential for sustainable exploitation of NTFP.
\end{abstract}

Keywords: Dense Ombrophilous Forest; biodiversity; PVSE; natural resources.

\section{INTRODUÇÃO}

Ao longo da história, as florestas têm sido úteis pelos produtos e benefícios que delas provêm, tanto para a subsistência quanto para o comércio (SANTOS et al., 2003). No início do século XX, o uso dos recursos florestais alavancou o desenvolvimento dos estados da região Sul do Brasil (Paraná, Santa Catarina e Rio Grande do Sul). O processo de geração de renda via venda da madeira pelo fornecimento de matéria-prima para construção civil atrelado à expansão da fronteira agrícola. Como estratégia, essa exploração esteve intimamente ligada à questão colonizadora. Os agricultores tradicionais do sul do Brasil incorporaram aos elementos da paisagem florestal uma forma de obter recursos para o consumo e suprir necessidades de renda durante o ano todo (CORADIN; SIMINSKI; REIS, 2011).

O desenvolvimento florestal tem se limitado

1 Biólogo, MSc., Doutorando do Programa de Pós-graduação em Ciências Ambientais, Universidade do Extremo Sul Catarinense, Av. Universitária, 1105, CEP 88806-000, Criciúma (SC).guielias1@hotmail.com

2 Biólogo, Dr., Professor do Programa de Pós-graduação em Ciências Ambientais, Universidade do Extremo Sul Catarinense, Av. Universitária, 1105, CEP 88806-000, Criciúma (SC).rsa@unesc.net 
à utilização de apenas um produto, a madeira. Essas perspectivas resultam em uso intensivo dos recursos madeireiros, em detrimento da constante desconsideração do restante do ecossistema florestal. Neste processo se gera perda iminente de importantes componentes da agregação de valor aos recursos naturais (SANTOS et al., 2003).

Os produtos florestais não madeireiros constituem um meio de subsistência para muitas comunidades, sendo também elementos significativos da economia rural e regional (VILLALOBOS; OCAMPO, 1997). Fazem parte de uma prática ancestral, economicamente viável de extração, que procura manter a estrutura e a funcionalidade da floresta (BALZON; SILVA; SANTOS, 2004).

O termo produto florestal não madeireiro (PFNM) se refere aos diferentes produtos vegetais e animais que se obtêm de ambientes florestais, que podem ser extraídos de florestas naturais, agroecossistemas e de árvores que crescem espontaneamente. Podem ter utilização doméstica, ser comercializados ou ter significado social, cultural ou religioso. Incluem frutas, fibras, sementes, plantas medicinais, aromáticas e apícolas, materiais para artesanato, entre outros. São bens de origem biológica diferentes da madeira, assim como serviços derivados das florestas e do uso das terras vinculadas a estes sistemas (WONG; THORNBER; BAKER, 2001; VANTOMME, 2001).

As definições existentes incluem explicitamente produtos provenientes de terras "com uso similar à floresta", enfatizando a colheita a partir de populações naturais como uma qualidade indispensável dos PFNM. Assume-se como premissa que uma espécie proveniente de um sistema de cultivo deixa de ser um PFNM para se incluído no processo de domesticação clássica (VILLALOBOS; OCAMPO, 1997).

Nepstad e Schwarzma (1992) salientam que, quando da extração de PFNM, a estrutura e a função da floresta não são alteradas e, na maioria dos casos, essa operação não envolve a degradação dos recursos naturais. Consideram os fatos que o uso desses produtos foi introduzido como estratégia de conservação e de desenvolvimento florestal. Os sistemas de produção dos PFNM estão inseridos em uma série de relações sociais, políticas e institucionais, não só econômicas e ecológicas (ALEXIADES; SHANLEY, 2004).

Vários estudos têm sugerido que o retorno econômico, em longo prazo, para o manejo adequado dos Produtos Florestais Não Madeireiros que se encontram em um hectare de floresta tropical sobrepõe os benefícios da produção de madeira ou da conversão agrícola da área (STATZ, 1997).

De acordo com Grimes; Loomis; Jahnige (1994), o manejo sustentável destes recursos pode prover benefícios para a população local enquanto promove, simultaneamente, a conservação dos ecossistemas florestais.

Os PFNM têm atraído considerável interesse global, nos últimos anos, devido ao crescente reconhecimento de sua contribuição aos objetivos socioeconômicos e ambientais. Incluir a conservação da diversidade biológica, uma vez que, ao mesmo tempo em que mantém a floresta conservada, permite melhoria do bem-estar da comunidade que nela vive (FAO, 1992, 1994, 1995).

Para que os PFNM sejam apresentados realmente como alternativas viáveis para estímulo à conservação e promoção de desenvolvimento de comunidades, precisa-se ainda esclarecer aspectos acerca do conhecimento botânico, ecológico e agronômico ou silvicultural das espécies fontes de PFNM. Com o intuito de contribuir com a conservação da biodiversidade, este estudo tem como objetivo indicar espécies arbóreas fontes de produtos florestais não madeireiros que poderão dar retorno socioeconômico e ambiental aos proprietários de áreas com remanescentes de Floresta Ombrófila Densa estudados no Sul de Santa Catarina.

\section{MATERIAIS E MÉTODOS}

O Sul do Estado de Santa Catarina ocupa área de $9.049 \mathrm{~km}^{2}$ (9,8\% da área total do Estado) que compreende 44 municípios, com população estimada de 900 mil habitantes (IBGE, 2013). As principais atividades econômicas são: a extração do carvão mineral, a agropecuária (cultivo de arroz e fumo, criação de frangos e produção de ovos), a indústria têxtil, a indústria cerâmica e a construção civil.

Os trabalhos selecionados para o presente estudo foram realizados em remanescentes de Floresta Ombrófila Densa, das Terras Baixas (MARTINS, 2010; PACHECO, 2010), Submontana (CITADINI-ZANETTE, 1995; SANTOS, 2003; MARTINS, 2005; REBELO, 2006; SILVA, 2006; PASETTO, 2008; COLONETTI et al., 2009; 
EMERICH, 2009) e Montana (MARTINS, 2010; BOSA, 2011) no Sul de Santa Catarina. Todos os levantamentos utilizaram o mesmo método de inclusão, ou seja, diâmetro a altura do peito (DAP) maior ou igual a $5 \mathrm{~cm}$.

O levantamento dos produtos florestais não madeireiros (PFNM) contemplou espécies arbóreas, selecionadas com base em sua frequência de ocorrência nos estudos $(\mathrm{F}=\mathrm{n} / \mathrm{N} \times 100)$, em que: $\mathrm{F}=$ frequência que a espécie é citada (\%), $\mathrm{n}=$ número de estudos nos quais consta a espécie $\mathrm{e}$ $\mathrm{N}=$ número total de estudos (12), e em sua densidade média $(\mathrm{Dm}=\mathrm{D} / \mathrm{N})$, sendo $\mathrm{Dm}=$ densidade média de cada espécie (indivíduos.ha ${ }^{-1}$ ), D = soma das densidades absolutas da espécie nos estudos e $\mathrm{N}$ = número total de estudos em que a espécie foi citada.

Foram selecionadas, para a composição da tabela do valor potencial de exploração sustentável (VPES), as espécies arbóreas que apresentaram frequência igual ou acima de $40 \%$ e densidade média igual ou acima de 5 indivíduos.ha ${ }^{-1}$. Deste modo, aquelas espécies que apresentaram VPES superior ou igual a 10 foram consideradas de maior importância para o uso como PFNM, consistindo nos mais indicados para uso da população do local.

Para cada uma das espécies-foco deste estudo foi realizada pesquisa exploratório-descritiva em várias fontes escritas e eletrônicas, buscandose informações sobre distribuição, abundância, dados ecológicos, agronômicos, fitoquímicos e farmacológicos, além de uso e aplicações como PFNM. Para tal fim, foram obtidas informações a partir de consulta às bases de dados disponíveis na Biblioteca Central da Universidade do Extremo Sul Catarinense, além de outros sites de busca. As palavras-chave empregadas nesse processo foram correspondentes aos nomes científicos das espécies e sinonímias, quando presentes.

Adicionalmente, dados sobre toxicidade e/ ou potencial de injúria sobre as espécies estudadas foram obtidos junto ao Poisonous Plant Database (FDA, 2012). As informações obtidas foram organizadas, a fim de facilitar o cálculo do VPES, como proposto por Ubessi-Macarini; Negrelle; Souza (2011), em que partem do princípio que o extrativismo sustentável deve ser capaz de manter a biodiversidade local, assim como gerar produtos para subsistência e comercialização, como indicado em FAO (1995). Desta forma, os seguintes indicativos, e respectivos valores $(0,1$ e 2$)$, foram organizados de maneira a facilitar o cálculo do VPES

As informações relativas a usos e aplicações foram sistematizadas de acordo com as categorias indicadas em FAO (1992), ou seja:

Ornamental: jardinagem, floricultura, decoração, arborização e/ou paisagismo; apícola (produção de mel pelas abelhas).

Forrageira: forragem para animais de criação.

Alimentícia e/ou aditivos: partes comestíveis utilizadas na alimentação humana, incluindo condimentos e temperos.

Medicinal: medicina popular e/ou produto bioquímico de interesse farmacêutico, tanto para tratamento de humanos quanto de uso veterinário.

Produto bioquímico: composto(s) químico(s) como tanino, corante, látex, goma, resina, óleo e toxina, entre outros de interesse farmacêutico ou químico-industrial.

Artesanato: confecções de utensílios e artefatos produzidos em escala artesanal, a partir de qualquer de suas partes, exceto tronco inteiro.

Fibra: cordaria, cestaria, confecção de peças do vestuário e chapéus, entre outros.

Ecológico: sementes e plântulas usadas em programas de reflorestamento ou recuperação de áreas degradadas, agrossilvicultura, cortina vegetal ou recurso para a fauna.

Outros usos: usos diversos, não referenciados nas categorias anteriores, como, por exemplo, o doméstico, para cobertura de casas, sombreamento de cultivos, enchimento de travesseiros, jogos, fins religiosos ou místicos.

Para calcular o VPES, os seguintes parâmetros foram considerados: parte usada, densidade, índices de regeneração e crescimento e conhecimento ecológico geral. A cada parâmetro foram designados valores normalizados $(0,1$ e 2). A ausência de informações recebeu o valor 0 (zero), que foi enfatizada, na escrita, pelo negrito. A somatória desses valores resultou nos VPES, que corresponderam aos valores crescentes de sustentabilidade de uso, conforme explicitado a seguir. Os valores de VPES igual ou superior a 10 (máximo $=14)$ foram designados como de alta potencialidade de exploração sustentável.

Para se chegar ao montante do cálculo do VPES, foram levados em consideração os seguintes itens proposto por Ubessi-Macarini; Negrelle; Souza (2011):

A) Parte usada da planta: 0 = ausência de informação; $0=$ alto nível de injúria ou que provoca 
a morte (planta inteira, tronco, casca, nó ou raiz); 1 = médio nível de injúria (flores, frutos, brotos, ramos ou sementes); 2 = nenhum nível de injúria ou mínimo (exsudados, resina, látex, seiva, goma, folhas).

B) Densidade de árvores ou abundância: $\mathbf{0}=$ ausência de informação; $0=$ baixa $(\leq 5$ indivíduos. ha $\left.^{-1}\right) ; 1=$ média $\left(6\right.$ a 15 indivíduos.ha $\left.^{-1}\right) ; 2=$ alta (> 15 indivíduos.ha $\left.{ }^{-1}\right)$.

C) Taxa de produção de sementes: 0 = ausência de informação; 0 = baixa ou irregular produção de sementes; 1 = moderada produção de sementes; 2 = alta produção de sementes.

D) Taxa de crescimento: $\mathbf{0}=$ ausência de informação; $0=$ crescimento natural lento; $1=$ crescimento natural moderado; $2=$ crescimento natural rápido.

E) Conhecimento ecológico geral: $\mathbf{0}=$ ausência de informação; $0=$ pouca informação disponível; 1 = alguma informação disponível; 2 = alto, incluindo informações sobre dinâmica populacional, biologia da reprodução e aspectos silviculturais.

F) Processamento: $\mathbf{0}=$ ausência de informação; $0=$ processamento dependente de equipamentos de alto custo; 1 = processamento dependente de equipamento de baixo custo;
$2=$ consumo in natura.

G) Injúria/toxicidade para humanos, durante a coleta, manuseio ou consumo, dentre outros: $\mathbf{0}=$ ausência de informação; $0=$ injúria/ toxicidade severa ou não controlável; $1=$ injúria/ toxicidade mediana ou controlável; 2 = nada consta, ou reconhecidamente sem injúria.

\section{RESULTADOS E DISCUSSÃO}

O levantamento dos produtos florestais não madeireiros (PFNM), para o cálculo do valor potencial de exploração sustentável (VPES), foi realizado para 79 espécies arbóreas, citando 191 usos, dentro das nove classes de uso, dentre 331 espécies levantadas nos 12 estudos. Ficou evidente a ausência de estudos fitossociológicos na maioria dos municípios (de 44 municípios, 36 não possuem estudos fitossociológicos concluídos), bem como, a dificuldade de caracterização de algumas espécies, quanto aos usos, pois algumas delas não constavam nas referências consultadas. O número de espécies arbóreas citadas nos estudos variou de 17 a 126 (Tabela 1).

Dentre as espécies selecionadas (Tabela 2) como as mais representativas (densidade e frequência), algumas se destacaram e foram

TABELA 1: Número de espécies citadas nos levantamentos florísticos e fitossociológicos e número de citações como fonte de produtos florestais não madeireiros (PFNM) na Floresta Ombrófila Densa em oito municípios do Sul de Santa Catarina.

TABLE 1: Number of species cited in floristic and phytosociological studies, and number of citations as a source of non-timber forest products (PFNM) in the Tropical Rain Forest in eight counties of Southern Santa Catarina.

\begin{tabular}{lccccc}
\hline \multirow{2}{*}{ Estudo } & Município & Formação florestal & Estádio & \multicolumn{2}{c}{ Número de espécies arbóreas } \\
\cline { 5 - 6 } & sucessional & Citadas & PFNM \\
\hline Bosa (2011) & Morro Grande & Montana & Avançado & 97 & 22 \\
Colonetti et al. (2009) & Siderópolis & Submontana & Avançado & 105 & 19 \\
Emerich (2009) & Turvo & Submontana & Avançado & 122 & 37 \\
Martins (2005) & Siderópolis & Submontana & Avançado & 95 & 18 \\
Martins (2010) & Araranguá & das Terras Baixas & Avançado & 17 & 9 \\
Martins (2010) & Timbé do Sul & Montana & Avançado & 124 & 21 \\
Pacheco (2010) & Criciúma & das Terras Baixas & Avançado & 116 & 33 \\
Pasetto (2008) & Siderópolis & Submontana & Avançado & 99 & 22 \\
Rebelo (2006) & Laguna & Submontana & Avançado & 98 & 8 \\
Santos (2003) & Siderópolis & Submontana & Médio & 77 & 27 \\
Silva (2006) & Criciúma & Submontana & Avançado & 126 & 28 \\
\hline
\end{tabular}


TABELA 2: Espécies arbóreas de Floresta Ombrófila Densa no Sul de Santa Catarina e respectivas informações relativas a usos e valor potencial de exploração sustentável (VPES). As colunas designadas por letras maiúsculas correspondem a valores relativos a: $\mathrm{A}=$ parte usada da planta; $\mathrm{B}=$ densidade (densidade média); $\mathrm{C}$ = produção de sementes; $\mathrm{D}=$ taxa de crescimento; $\mathrm{E}=$ demanda de processamento; $\mathrm{F}$ = conhecimento ecológico geral disponível; $\mathrm{G}=$ potencial de injúria. $\mathrm{Dm}=$ densidade média (indivíduos.ha ${ }^{-1}$ ) e $\mathrm{FA}=$ frequência absoluta $(\%)$, com que a espécie ocorre.

TABLE 2: Tree species rain forest in southern Santa Catarina and their information on uses and potential value of sustainable (VPES). The columns designated by capital letters correspond to the relative values: $\mathrm{A}=$ used part of the plant, $\mathrm{B}=$ density (mean density), $\mathrm{C}=$ seed production, $\mathrm{D}$ $=$ growth rate, $\mathrm{E}=$ processing demand, $\mathrm{F}=$ overall ecological knowledge available; $\mathrm{G}=$ level of injury or toxicity. $\mathrm{Dm}=$ average density (individuals.ha ${ }^{-1}$ ) and $\mathrm{FA}=$ absolute frequency $(\%)$ with which the species occurs.

\begin{tabular}{|c|c|c|c|c|c|c|c|c|c|c|c|c|}
\hline Espécie & Usos & $\begin{array}{c}\text { Parte } \\
\text { utilizada }\end{array}$ & A & B & $\mathrm{C}$ & $\mathrm{D}$ & $\mathrm{E}$ & $\mathrm{F}$ & G & VPES & Dm & FA \\
\hline \multirow{2}{*}{ Aegiphyla sellowiana Cham. } & ecológico & sementes & 1 & 1 & 2 & 2 & 2 & 2 & 2 & 12 & \multirow{2}{*}{8} & \multirow{2}{*}{58} \\
\hline & ornamental & sementes & 1 & 1 & 2 & 2 & 2 & 2 & 2 & 12 & & \\
\hline \multirow{2}{*}{ Aiouea saligna Meisn. } & ecológico & sementes & 1 & 1 & 2 & 0 & 2 & 2 & 2 & 10 & \multirow{2}{*}{10} & \multirow{2}{*}{67} \\
\hline & ornamental & sementes & 1 & 1 & 2 & 0 & 2 & 2 & 2 & 10 & & \\
\hline \multirow{2}{*}{ Alchornea triplinervia (Spreng.) Müll.Arg. } & apícola & flores & 1 & 2 & 1 & 2 & 1 & 0 & 2 & 9 & \multirow{2}{*}{27} & \multirow{2}{*}{100} \\
\hline & ornamental & semente & 1 & 2 & 1 & 2 & 2 & 0 & 2 & 10 & & \\
\hline \multirow{5}{*}{$\begin{array}{l}\text { Allophylus edulis (St.-Hil., Cambess. et } \\
\text { Juss.) Radlk. }\end{array}$} & apícola & flores & 1 & 2 & 2 & 2 & 2 & 2 & 2 & 13 & \multirow{5}{*}{19} & \multirow{5}{*}{67} \\
\hline & ecológico & sementes & 1 & 2 & 2 & 2 & 2 & 2 & 2 & 13 & & \\
\hline & medicinal & folhas & 2 & 2 & 2 & 2 & 2 & 2 & 2 & 14 & & \\
\hline & ornamental & sementes & 1 & 2 & 2 & 2 & 2 & 2 & 2 & 13 & & \\
\hline & $\begin{array}{l}\text { produto } \\
\text { bioquímico }\end{array}$ & folhas & 2 & 2 & 2 & 2 & 0 & 2 & 2 & 12 & & \\
\hline \multirow{5}{*}{ Annona neosericea H.Rainer } & alimentícia & frutos & 1 & 1 & 2 & 1 & 2 & 2 & 2 & 11 & \multirow{5}{*}{14} & \multirow{5}{*}{83} \\
\hline & artesanato & cascas & 0 & 1 & 2 & 1 & 2 & 2 & 2 & 10 & & \\
\hline & ecológico & sementes & 1 & 1 & 2 & 1 & 2 & 2 & 2 & 11 & & \\
\hline & fibra & casca & 0 & 1 & 2 & 1 & 2 & 2 & 2 & 10 & & \\
\hline & ornamental & sementes & 1 & 1 & 2 & 1 & 2 & 2 & 2 & 11 & & \\
\hline \multirow{2}{*}{ Annona rugulosa (Schltdl.) H.Rainer } & ecológico & sementes & 1 & 1 & $\mathbf{0}$ & 0 & 2 & 0 & $\mathbf{0}$ & 4 & \multirow{2}{*}{6} & \multirow{2}{*}{50} \\
\hline & ornamental & sementes & 1 & 1 & $\mathbf{0}$ & $\mathbf{0}$ & 2 & 0 & $\mathbf{0}$ & 4 & & \\
\hline \multirow{4}{*}{ Aspidosperma parvifolium A.DC. } & apícola & flores & 1 & 2 & 2 & 0 & 1 & 2 & 2 & 10 & \multirow{4}{*}{16} & \multirow{4}{*}{67} \\
\hline & ecológico & sementes & 1 & 2 & 2 & 0 & 2 & 2 & 2 & 11 & & \\
\hline & medicinal & cascas & 0 & 2 & 2 & 0 & 1 & 2 & 2 & 9 & & \\
\hline & ornamental & sementes & 1 & 2 & 2 & 0 & 2 & 2 & 2 & 11 & & \\
\hline \multirow{2}{*}{ Banara parviflora Benth. } & ecológico & sementes & 1 & 1 & 2 & 1 & 2 & 2 & 2 & 11 & \multirow{2}{*}{9} & \multirow{2}{*}{42} \\
\hline & ornamental & sementes & 1 & 1 & 2 & 1 & 2 & 2 & 2 & 11 & & \\
\hline \multirow{4}{*}{ Bathysa australis (St.-Hil.) Benth. et Hook. } & apícola & flores & 1 & 2 & 2 & 1 & 1 & 2 & 2 & 11 & \multirow{4}{*}{45} & \multirow{4}{*}{75} \\
\hline & ecológico & sementes & 1 & 2 & 2 & 1 & 2 & 2 & 2 & 12 & & \\
\hline & medicinal & cascas & 0 & 2 & 2 & 1 & 0 & 2 & 2 & 9 & & \\
\hline & ornamental & sementes & 1 & 2 & 2 & 1 & 2 & 2 & 2 & 12 & & \\
\hline
\end{tabular}


TABELA 2: Continuação...

TABLE 2: Continued...

\begin{tabular}{|c|c|c|c|c|c|c|c|c|c|c|c|c|}
\hline Espécie & Usos & $\begin{array}{c}\text { Parte } \\
\text { utilizada }\end{array}$ & A & $\mathrm{B}$ & $\mathrm{C}$ & $\mathrm{D}$ & $\mathrm{E}$ & $\mathrm{F}$ & G & VPES & $\mathrm{Dm}$ & FA \\
\hline \multirow{2}{*}{ Brosimum glaziovii Taub. } & ecológico & sementes & 1 & 1 & 1 & $\mathbf{0}$ & 2 & 0 & $\mathbf{0}$ & 5 & 8 & 58 \\
\hline & ornamental & semente & 1 & 1 & 1 & $\mathbf{0}$ & 2 & 0 & $\mathbf{0}$ & 5 & & \\
\hline \multirow{3}{*}{ Byrsonima ligustrifolia St.-Hil. } & alimentícia & frutos & 1 & 2 & $\mathbf{0}$ & $\mathbf{0}$ & $\mathbf{0}$ & 2 & $\mathbf{0}$ & 5 & & \\
\hline & apícola & flores & 1 & 2 & $\mathbf{0}$ & $\mathbf{0}$ & $\mathbf{0}$ & 1 & $\mathbf{0}$ & 4 & 19 & 58 \\
\hline & ecológico & sementes & 1 & 2 & $\mathbf{0}$ & $\mathbf{0}$ & $\mathbf{0}$ & 2 & $\mathbf{0}$ & 5 & & \\
\hline \multirow{6}{*}{ Cabralea canjerana (Vell.) Mart. } & alimentícia & frutos & 1 & 2 & 2 & 0 & 2 & 2 & 2 & 11 & \multirow{6}{*}{34} & \multirow{6}{*}{91} \\
\hline & artesanato & cascas & 0 & 2 & 2 & 0 & 1 & 2 & 2 & 9 & & \\
\hline & ecológico & sementes & 1 & 2 & 2 & 0 & 2 & 2 & 2 & 11 & & \\
\hline & medicinal & cascas & 0 & 2 & 2 & 0 & 0 & 2 & 2 & 8 & & \\
\hline & ornamental & sementes & 1 & 2 & 2 & 0 & 2 & 2 & 2 & 11 & & \\
\hline & $\begin{array}{c}\text { produto } \\
\text { bioquímico }\end{array}$ & frutos & 1 & 2 & 2 & 0 & 1 & 2 & 2 & 10 & & \\
\hline Calyptranthes grandifolia O.Berg & ecológico & sementes & 1 & 1 & 1 & $\mathbf{0}$ & 2 & $\mathbf{0}$ & $\mathbf{0}$ & 5 & 6 & 50 \\
\hline Calyptranthes lucida Mart. ex DC. & ecológico & sementes & 1 & 2 & 1 & $\mathbf{0}$ & 2 & 0 & $\mathbf{0}$ & 6 & 20 & 50 \\
\hline \multirow{3}{*}{ Casearia sylvestris Sw. } & medicinal & folhas & 2 & 2 & 2 & 1 & 0 & 2 & 2 & 11 & \multirow{3}{*}{48} & \multirow{3}{*}{83} \\
\hline & ornamental & sementes & 1 & 2 & 2 & 1 & 2 & 2 & 2 & 12 & & \\
\hline & $\begin{array}{c}\text { produto } \\
\text { bioquímico }\end{array}$ & folhas & 2 & 2 & 2 & 1 & 1 & 2 & 2 & 12 & & \\
\hline \multirow{6}{*}{ Cedrela fissilis Vell. } & alimentícia & frutos & 1 & 1 & 2 & 0 & 2 & 2 & 2 & 10 & \multirow{6}{*}{10} & \multirow{6}{*}{91} \\
\hline & apícola & flores & 1 & 1 & 2 & 0 & 1 & 2 & 2 & 9 & & \\
\hline & forrageira & folhas & 2 & 1 & 2 & 0 & 2 & 2 & 2 & 11 & & \\
\hline & medicinal & cascas & 0 & 1 & 2 & 0 & 0 & 2 & 2 & 7 & & \\
\hline & ornamental & sementes & 2 & 1 & 2 & 0 & 2 & 2 & 2 & 11 & & \\
\hline & $\begin{array}{c}\text { produto } \\
\text { bioquímico }\end{array}$ & cascas & 0 & 1 & 2 & 0 & 0 & 2 & 2 & 7 & & \\
\hline Chrysophyllum inornatum Mart. & ecológico & sementes & 1 & 2 & 1 & $\mathbf{0}$ & 2 & $\mathbf{0}$ & $\mathbf{0}$ & 6 & 16 & 75 \\
\hline \multirow{2}{*}{ Chrysophyllum viride Mart. et Eichl. } & alimentícia & frutos & 1 & 1 & 1 & $\mathbf{0}$ & 2 & $\mathbf{0}$ & $\mathbf{0}$ & 5 & \multirow{2}{*}{6} & \multirow{2}{*}{58} \\
\hline & ecológico & sementes & 1 & 1 & 1 & $\mathbf{0}$ & 2 & $\mathbf{0}$ & $\mathbf{0}$ & 5 & & \\
\hline Cinnamomum glaziovii (Mez) Kosterm. & ecológico & sementes & 1 & 1 & 1 & $\mathbf{0}$ & 2 & $\mathbf{0}$ & $\mathbf{0}$ & 5 & 7 & 67 \\
\hline Cordia silvestris Fresen. & ecológico & sementes & 1 & 1 & 1 & $\mathbf{0}$ & 2 & $\mathbf{0}$ & $\mathbf{0}$ & 5 & 6 & 42 \\
\hline \multirow{2}{*}{ Coussapoa microcarpa (Schott.) Rizz. } & ecológico & sementes & 1 & 1 & 2 & $\mathbf{0}$ & 2 & 0 & $\mathbf{0}$ & 6 & \multirow{2}{*}{7} & \multirow{2}{*}{75} \\
\hline & ornamental & sementes & 1 & 1 & 2 & $\mathbf{0}$ & 2 & 0 & $\mathbf{0}$ & 6 & & \\
\hline \multirow{3}{*}{ Cupania vernalis Cambess. } & medicinal & folhas & 2 & 1 & 2 & 0 & 0 & 0 & 2 & 7 & \multirow{3}{*}{9} & \multirow{3}{*}{50} \\
\hline & ornamental & sementes & 1 & 1 & 2 & 0 & 2 & 0 & 2 & 8 & & \\
\hline & $\begin{array}{c}\text { produto } \\
\text { bioquímico }\end{array}$ & cascas & 0 & 1 & 2 & 0 & 0 & 0 & 2 & 5 & & \\
\hline \multirow{2}{*}{ Duguetia lanceolata St.-Hil. } & ecológico & sementes & 1 & 1 & 2 & 0 & 2 & 2 & 2 & 10 & \multirow{2}{*}{10} & \multirow{2}{*}{42} \\
\hline & ornamental & sementes & 1 & 1 & 2 & 0 & 2 & 2 & 2 & 10 & & \\
\hline Endlicheria paniculata (Spreng.) Macbr. & ecológico & sementes & 1 & 1 & 1 & 1 & 2 & 2 & 2 & 10 & 6 & 91 \\
\hline
\end{tabular}


TABELA 2: Continuação...

TABLE 2: Continued...

\begin{tabular}{|c|c|c|c|c|c|c|c|c|c|c|c|c|}
\hline Espécie & Usos & $\begin{array}{c}\text { Parte } \\
\text { utilizada }\end{array}$ & A & B & $\mathrm{C}$ & $\mathrm{D}$ & $\mathrm{E}$ & $\mathrm{F}$ & G & VPES & $\mathrm{Dm}$ & FA \\
\hline Esenbeckia grandiflora Mart. & ornamental & sementes & 1 & 1 & 1 & 1 & 2 & 2 & 2 & 10 & 10 & 75 \\
\hline \multirow{2}{*}{ Eugenia multicostata Legr. } & ecológico & sementes & 1 & 1 & 1 & 1 & 2 & 2 & 2 & 10 & \multirow{2}{*}{6} & \multirow{2}{*}{42} \\
\hline & ornamental & sementes & 1 & 1 & 1 & 1 & 2 & 2 & 2 & 10 & & \\
\hline \multirow{7}{*}{ Euterpe edulis Mart. } & alimentícia & frutos & 1 & 2 & 2 & 1 & 2 & 2 & 2 & 12 & \multirow{7}{*}{261} & \multirow{7}{*}{83} \\
\hline & artesanato & sementes & 1 & 2 & 2 & 1 & 2 & 2 & 2 & 12 & & \\
\hline & ecológico & sementes & 1 & 2 & 2 & 1 & 2 & 2 & 2 & 12 & & \\
\hline & fibra & folhas & 2 & 2 & 2 & 1 & 2 & 2 & 2 & 13 & & \\
\hline & forrageira & folhas & 2 & 2 & 2 & 1 & 2 & 2 & 2 & 13 & & \\
\hline & medicinal & frutos & 1 & 2 & 2 & 1 & 2 & 2 & 2 & 12 & & \\
\hline & ornamental & sementes & 1 & 2 & 2 & 1 & 2 & 2 & 2 & 12 & & \\
\hline \multirow{2}{*}{$\begin{array}{l}\text { Faramea montevidensis (Cham. et Schltdl.) } \\
\text { DC. }\end{array}$} & ecológico & sementes & 1 & 2 & 1 & 0 & 2 & 0 & 0 & 6 & \multirow{2}{*}{33} & \multirow{2}{*}{58} \\
\hline & ornamental & sementes & 1 & 2 & 1 & 0 & 2 & 0 & $\mathbf{0}$ & 6 & & \\
\hline \multirow{4}{*}{$\begin{array}{l}\text { Garcinia gardneriana (Planch. et Triana) } \\
\text { Zappi }\end{array}$} & alimentícia & frutos & 1 & 1 & 2 & 2 & 2 & 2 & 2 & 12 & \multirow{4}{*}{13} & \multirow{4}{*}{67} \\
\hline & ecológico & sementes & 1 & 1 & 2 & 2 & 2 & 2 & 2 & 12 & & \\
\hline & medicinal & folhas & 2 & 1 & 2 & 2 & 2 & 2 & 2 & 13 & & \\
\hline & ornamental & sementes & 1 & 1 & 2 & 2 & 2 & 2 & 2 & 12 & & \\
\hline Guapira opposita (Vell.) Reitz & ecológico & sementes & 1 & 2 & 1 & 1 & 2 & 2 & 2 & 11 & 26 & 83 \\
\hline Guarea macrophylla Vahl & ecológico & sementes & 1 & 2 & $\mathbf{0}$ & $\mathbf{0}$ & 2 & $\mathbf{0}$ & $\mathbf{0}$ & 5 & 19 & 67 \\
\hline Guatteria australis St.-Hil. & ecológico & sementes & 1 & 2 & $\mathbf{0}$ & $\mathbf{0}$ & 2 & $\mathbf{0}$ & $\mathbf{0}$ & 5 & 17 & 58 \\
\hline Gymnanthes concolor (Spreng.) Müll.Arg. & ecológico & sementes & 1 & 2 & $\mathbf{0}$ & $\mathbf{0}$ & 2 & $\mathbf{0}$ & $\mathbf{0}$ & 5 & 48 & 58 \\
\hline \multirow{2}{*}{ Heisteria silvianii Schwacke Schwacke } & ecológico & sementes & 1 & 1 & $\mathbf{0}$ & $\mathbf{0}$ & 2 & $\mathbf{0}$ & $\mathbf{0}$ & 4 & \multirow{2}{*}{9} & \multirow{2}{*}{58} \\
\hline & ornamental & sementes & 1 & 1 & $\mathbf{0}$ & $\mathbf{0}$ & 2 & $\mathbf{0}$ & $\mathbf{0}$ & 4 & & \\
\hline \multirow{3}{*}{ Hieronyma alchorneoides Fr.Allem. } & apícola & flores & 1 & 2 & 2 & 0 & 1 & 2 & 2 & 10 & \multirow{3}{*}{22} & \multirow{3}{*}{75} \\
\hline & ecológico & sementes & 1 & 2 & 2 & 0 & 2 & 2 & 2 & 11 & & \\
\hline & ornamental & sementes & 1 & 2 & 2 & 0 & 2 & 2 & 2 & 11 & & \\
\hline \multirow{2}{*}{ Hirtella hebeclada Moric. ex DC. } & ecológico & sementes & 1 & 2 & 1 & 1 & 2 & 2 & 2 & 11 & \multirow{2}{*}{17} & \multirow{2}{*}{75} \\
\hline & ornamental & sementes & 1 & 2 & 1 & 1 & 2 & 2 & 2 & 11 & & \\
\hline \multirow{7}{*}{ Ilex paraguariensis St.-Hil. } & alimentícia & folhas & 2 & 1 & $\mathbf{0}$ & $\mathbf{0}$ & 1 & 2 & 2 & 8 & \multirow{7}{*}{10} & \\
\hline & apícola & flores & 1 & 1 & $\mathbf{0}$ & $\mathbf{0}$ & 1 & 2 & 2 & 7 & & \\
\hline & ecológico & sementes & 1 & 1 & $\mathbf{0}$ & $\mathbf{0}$ & 2 & 2 & 2 & 8 & & \\
\hline & forrageira & folhas & 2 & 1 & $\mathbf{0}$ & $\mathbf{0}$ & 2 & 2 & 2 & 9 & & 42 \\
\hline & medicinal & folhas & 2 & 1 & $\mathbf{0}$ & $\mathbf{0}$ & 1 & 2 & 2 & 8 & & 42 \\
\hline & ornamental & sementes & 1 & 1 & $\mathbf{0}$ & $\mathbf{0}$ & 2 & 2 & 2 & 8 & & \\
\hline & $\begin{array}{c}\text { produto } \\
\text { bioquímico }\end{array}$ & folhas & 2 & 1 & $\mathbf{0}$ & $\mathbf{0}$ & 1 & 2 & 2 & 8 & & \\
\hline & alimentícia & frutos & 1 & 1 & 2 & 1 & 2 & 2 & 2 & 11 & & \\
\hline Inga sessilis (Vell.) Mart. & apícola & flores & 1 & 1 & 2 & 1 & 1 & 2 & 2 & 10 & 6 & 67 \\
\hline & ecológico & sementes & 1 & 1 & 2 & 1 & 2 & 2 & 2 & 11 & & \\
\hline & ecológico & sementes & 1 & 2 & 2 & 2 & 2 & 2 & 2 & 13 & 68 & 58 \\
\hline sacaraverata & ornamental & sementes & 1 & 2 & 2 & 2 & 2 & 2 & 2 & 13 & 00 & \\
\hline
\end{tabular}

Continua... 
TABELA 2: Continuação...

TABLE 2: Continued...

\begin{tabular}{|c|c|c|c|c|c|c|c|c|c|c|c|c|}
\hline Espécie & Usos & $\begin{array}{c}\text { Parte } \\
\text { utilizada }\end{array}$ & A & $\mathrm{B}$ & $\mathrm{C}$ & $\mathrm{D}$ & $\mathrm{E}$ & $\mathrm{F}$ & G & VPES & $\mathrm{Dm}$ & FA \\
\hline \multirow[b]{2}{*}{ Lamanonia ternata Vell. } & ecológico & sementes & 1 & 2 & 2 & 1 & 2 & 2 & 2 & 12 & \multirow[b]{2}{*}{22} & \multirow[b]{2}{*}{50} \\
\hline & $\begin{array}{c}\text { produto } \\
\text { bioquímico }\end{array}$ & cascas & 0 & 2 & 2 & 1 & 0 & 2 & 2 & 9 & & \\
\hline \multirow{6}{*}{ Luehea divaricata Mart. } & apícola & flores & 1 & 1 & 2 & 1 & 1 & 2 & 2 & 10 & \multirow{6}{*}{9} & \multirow{6}{*}{67} \\
\hline & artesanato & casca & 0 & 1 & 2 & 1 & 1 & 2 & 2 & 9 & & \\
\hline & ecológico & sementes & 1 & 1 & 2 & 1 & 2 & 2 & 2 & 11 & & \\
\hline & medicinal & folhas & 2 & 1 & 2 & 1 & 1 & 2 & 2 & 11 & & \\
\hline & ornamental & sementes & 1 & 1 & 2 & 1 & 2 & 2 & 2 & 11 & & \\
\hline & $\begin{array}{c}\text { produto } \\
\text { bioquímico }\end{array}$ & cascas & 0 & 1 & 2 & 1 & 0 & 2 & 2 & 8 & & \\
\hline \multirow{5}{*}{ Machaerium stipitatum Vogel } & apícola & flores & 1 & 1 & $\mathbf{0}$ & $\mathbf{0}$ & 1 & 0 & $\mathbf{0}$ & 3 & \multirow{5}{*}{6} & \multirow{5}{*}{42} \\
\hline & ecológico & sementes & 1 & 1 & $\mathbf{0}$ & $\mathbf{0}$ & 2 & 0 & $\mathbf{0}$ & 4 & & \\
\hline & forrageira & folhas & 2 & 1 & $\mathbf{0}$ & $\mathbf{0}$ & 2 & 0 & $\mathbf{0}$ & 5 & & \\
\hline & medicinal & folhas & 2 & 1 & $\mathbf{0}$ & $\mathbf{0}$ & 2 & 0 & $\mathbf{0}$ & 5 & & \\
\hline & ornamental & sementes & 1 & 1 & $\mathbf{0}$ & $\mathbf{0}$ & 2 & 0 & $\mathbf{0}$ & 4 & & \\
\hline \multirow{2}{*}{ Magnolia ovata (St.-Hil.) Spreng. } & artesanato & sementes & 1 & 1 & $\mathbf{0}$ & $\mathbf{0}$ & 2 & 0 & $\mathbf{0}$ & 4 & \multirow{2}{*}{15} & \multirow{2}{*}{75} \\
\hline & ecológico & sementes & 1 & 1 & $\mathbf{0}$ & $\mathbf{0}$ & 2 & 0 & $\mathbf{0}$ & 4 & & \\
\hline Marlierea silvatica (Gardner) Kiaersk. & ecológico & sementes & 1 & 2 & $\mathbf{0}$ & $\mathbf{0}$ & 2 & 0 & $\mathbf{0}$ & 5 & 21 & 50 \\
\hline \multirow{2}{*}{ Matayba guianensis Aubl. } & ecológico & sementes & 1 & 2 & 1 & 1 & 2 & 2 & 2 & 11 & \multirow{2}{*}{50} & \multirow{2}{*}{83} \\
\hline & ornamental & sementes & 1 & 2 & 1 & 1 & 2 & 2 & 2 & 11 & & \\
\hline \multirow{2}{*}{ Meliosma sellowii Urban } & ecológico & sementes & 1 & 2 & 2 & 2 & 2 & 2 & 2 & 13 & \multirow{2}{*}{26} & \multirow{2}{*}{58} \\
\hline & ornamental & sementes & 1 & 2 & 2 & 2 & 2 & 2 & 2 & 13 & & \\
\hline Miconia cabucu Hoehne & ecológico & sementes & 1 & 1 & $\mathbf{0}$ & $\mathbf{0}$ & 2 & 0 & $\mathbf{0}$ & 4 & 12 & 58 \\
\hline Mollinedia schottiana (Spreng.) Perkins & medicinal & folhas & 2 & 1 & $\mathbf{0}$ & $\mathbf{0}$ & 1 & 0 & $\mathbf{0}$ & 4 & 14 & 67 \\
\hline \multirow{2}{*}{ Myrcia glabra (Berg) Legr. } & ecológico & sementes & 1 & 1 & $\mathbf{0}$ & $\mathbf{0}$ & 2 & 0 & $\mathbf{0}$ & 4 & \multirow{2}{*}{7} & \multirow{2}{*}{50} \\
\hline & ornamental & sementes & 1 & 1 & $\mathbf{0}$ & $\mathbf{0}$ & 2 & 0 & $\mathbf{0}$ & 4 & & \\
\hline Myrcia pubipetala Miq. & ecológico & sementes & 1 & 2 & $\mathbf{0}$ & $\mathbf{0}$ & 2 & 0 & $\mathbf{0}$ & 5 & 22 & 75 \\
\hline Myrcia richardiana (O.Berg) Kiaersk. & ecológico & sementes & 1 & 1 & $\mathbf{0}$ & $\mathbf{0}$ & 2 & 0 & $\mathbf{0}$ & 4 & 8 & 50 \\
\hline \multirow{2}{*}{ Myrcia splendens (Sw.) DC. } & ecológico & sementes & 1 & 2 & $\mathbf{0}$ & $\mathbf{0}$ & 1 & 0 & $\mathbf{0}$ & 4 & \multirow[t]{2}{*}{46} & 75 \\
\hline & medicinal & folhas & 2 & 2 & $\mathbf{0}$ & $\mathbf{0}$ & 1 & 0 & $\mathbf{0}$ & 5 & & \\
\hline & ecológico & sementes & 1 & 1 & $\mathbf{0}$ & $\mathbf{0}$ & 2 & 0 & $\mathbf{0}$ & 4 & 14 & 66 \\
\hline Myrcta lyucensts NTaersk. & ornamental & sementes & 1 & 1 & $\mathbf{0}$ & $\mathbf{0}$ & 2 & 0 & $\mathbf{0}$ & 4 & 14 & 00 \\
\hline Myrciaria floribunda (West ex Willd.) Berg & ecológico & sementes & 1 & 1 & $\mathbf{0}$ & $\mathbf{0}$ & 2 & 0 & $\mathbf{0}$ & 4 & 9 & 83 \\
\hline & apícola & flores & 1 & 1 & 2 & 1 & 1 & 2 & 2 & 10 & & \\
\hline Myrsine coriacea (Sw.) R.Br. & ecológico & sementes & 1 & 1 & 2 & 1 & 2 & 2 & 2 & 11 & 6 & 50 \\
\hline & ornamental & sementes & 1 & 1 & 2 & 1 & 2 & 2 & 2 & 11 & & \\
\hline Myrsine umbellata Mart. & ecológico & sementes & 1 & 1 & 2 & 1 & 2 & 2 & 2 & 11 & 12 & 75 \\
\hline & ecológico & sementes & 1 & 2 & 2 & 0 & 2 & 2 & 2 & 11 & 17 & 58 \\
\hline svectanar memor anacea (sw.) Unseo. & ornamental & sementes & 1 & 2 & 2 & 0 & 2 & 2 & 2 & 11 & & \\
\hline & ecológico & sementes & 1 & 2 & 2 & 0 & 2 & 2 & 2 & 11 & 17 & 67 \\
\hline & ornamental & sementes & 1 & 2 & 2 & 0 & 2 & 2 & 2 & 11 & & 01 \\
\hline
\end{tabular}


TABELA 2: Continuação...

TABLE 2: Continued...

\begin{tabular}{|c|c|c|c|c|c|c|c|c|c|c|c|c|}
\hline Espécie & Usos & $\begin{array}{c}\text { Parte } \\
\text { utilizada }\end{array}$ & A & B & $\mathrm{C}$ & $\mathrm{D}$ & $\mathrm{E}$ & $\mathrm{F}$ & G & VPES & $\mathrm{Dm}$ & FA \\
\hline \multirow[b]{2}{*}{ Ocotea catharinensis $\mathrm{Mez}$} & ecológico & sementes & 1 & 1 & 2 & 0 & 2 & 2 & 2 & 10 & \multirow[b]{2}{*}{11} & \multirow[b]{2}{*}{42} \\
\hline & $\begin{array}{c}\text { produto } \\
\text { bioquímico }\end{array}$ & cascas & 0 & 1 & 2 & 0 & 0 & 2 & 2 & 7 & & \\
\hline \multirow{2}{*}{ Ocotea indecora (Schott) Mez } & ecológico & sementes & 1 & 1 & $\mathbf{0}$ & $\mathbf{0}$ & 2 & 0 & $\mathbf{0}$ & 4 & \multirow{2}{*}{8} & \multirow{2}{*}{58} \\
\hline & ornamental & sementes & 1 & 1 & $\mathbf{0}$ & $\mathbf{0}$ & 2 & 0 & $\mathbf{0}$ & 4 & & \\
\hline \multirow{4}{*}{ Ocotea puberula (Rich.) Nees } & ecológico & sementes & 1 & 1 & 2 & 1 & 2 & 2 & 2 & 11 & \multirow{4}{*}{10} & \multirow{4}{*}{58} \\
\hline & forrageira & folhas & 2 & 1 & 2 & 1 & 2 & 2 & 2 & 12 & & \\
\hline & medicinal & folhas & 2 & 1 & 2 & 1 & 1 & 2 & 2 & 11 & & \\
\hline & ornamental & sementes & 1 & 1 & 2 & 1 & 2 & 2 & 2 & 11 & & \\
\hline Ocotea silvestris Vattimo & ecológico & sementes & 1 & 1 & $\mathbf{0}$ & $\mathbf{0}$ & 2 & 0 & $\mathbf{0}$ & 4 & 6 & 42 \\
\hline Ocotea urbaniana Mez & ecológico & sementes & 1 & 1 & $\mathbf{0}$ & $\mathbf{0}$ & 2 & 0 & $\mathbf{0}$ & 4 & 6 & 50 \\
\hline \multirow{2}{*}{ Pera glabrata (Schott) Poepp. ex Baill. } & ecológico & sementes & 1 & 1 & $\mathbf{0}$ & $\mathbf{0}$ & 2 & 0 & $\mathbf{0}$ & 4 & \multirow{2}{*}{6} & \multirow{2}{*}{67} \\
\hline & ornamental & sementes & 1 & 1 & $\mathbf{0}$ & $\mathbf{0}$ & 2 & 0 & $\mathbf{0}$ & 4 & & \\
\hline Piptocarpha tomentosa Baker & ecológico & sementes & 1 & 1 & $\mathbf{0}$ & $\mathbf{0}$ & 2 & 0 & $\mathbf{0}$ & 4 & 12 & 50 \\
\hline \multirow{2}{*}{$\begin{array}{l}\text { Posoqueria latifolia (Rudge) Roem. et } \\
\text { Schult. }\end{array}$} & ecológico & sementes & 1 & 1 & 2 & 1 & 2 & 2 & 2 & 11 & \multirow{2}{*}{14} & \multirow{2}{*}{75} \\
\hline & ornamental & sementes & 1 & 1 & 2 & 1 & 2 & 2 & 2 & 11 & & \\
\hline Protium kleinii Cuatrec. & ecológico & sementes & 1 & 1 & $\mathbf{0}$ & $\mathbf{0}$ & 2 & 0 & $\mathbf{0}$ & 4 & 10 & 58 \\
\hline \multirow{2}{*}{$\begin{array}{l}\text { Pseudobombax grandiflorum (Cav.) } \\
\text { A.Robyns }\end{array}$} & ecológico & sementes & 1 & 1 & 1 & 1 & 2 & 2 & 2 & 10 & \multirow{2}{*}{7} & \multirow{2}{*}{50} \\
\hline & ornamental & sementes & 1 & 1 & 1 & 1 & 2 & 2 & 2 & 10 & & \\
\hline \multirow{7}{*}{ Psidium cattleianum Sabine } & alimentícia & frutos & 1 & 2 & 2 & 1 & 2 & 2 & 2 & 12 & \multirow{7}{*}{16} & \multirow{7}{*}{42} \\
\hline & apícola & flores & 1 & 2 & 2 & 1 & 2 & 2 & 2 & 12 & & \\
\hline & artesanato & sementes & 1 & 2 & 2 & 1 & 2 & 2 & 2 & 12 & & \\
\hline & ecológico & sementes & 1 & 2 & 2 & 1 & 2 & 2 & 2 & 12 & & \\
\hline & medicinal & folhas & 2 & 2 & 2 & 1 & 2 & 2 & 2 & 13 & & \\
\hline & ornamental & sementes & 1 & 2 & 2 & 1 & 2 & 2 & 2 & 12 & & \\
\hline & $\begin{array}{l}\text { produto } \\
\text { bioquímico }\end{array}$ & cascas & 0 & 2 & 2 & 1 & 0 & 2 & 2 & 9 & & \\
\hline Psychotria suterella Müll.Arg. & ecológico & sementes & 1 & & $\mathbf{0}$ & $\mathbf{0}$ & 2 & 0 & $\mathbf{0}$ & 3 & 40 & 67 \\
\hline \multirow{4}{*}{ Roupala brasiliensis Klotzsch } & apícola & flores & 1 & 1 & $\mathbf{0}$ & $\mathbf{0}$ & 1 & 0 & $\mathbf{0}$ & 3 & \multirow{4}{*}{9} & \\
\hline & ecológico & sementes & 1 & 1 & $\mathbf{0}$ & $\mathbf{0}$ & 1 & 0 & $\mathbf{0}$ & 3 & & $42+2$ \\
\hline & medicinal & cascas & 0 & 1 & $\mathbf{0}$ & $\mathbf{0}$ & 0 & 0 & $\mathbf{0}$ & 2 & & 42 \\
\hline & ornamental & sementes & 1 & 1 & $\mathbf{0}$ & $\mathbf{0}$ & 1 & 0 & $\mathbf{0}$ & 3 & & \\
\hline Rudgea jasminoides (Cham.) Müll.Arg. & ornamental & sementes & 1 & 2 & $\mathbf{0}$ & $\mathbf{0}$ & 2 & 0 & $\mathbf{0}$ & 5 & 49 & 75 \\
\hline $\begin{array}{l}\text { Schefflera morototoni (Aubl.) Maguire, } \\
\text { Steyem. et Frodin }\end{array}$ & ecológico & sementes & 1 & 1 & $\mathbf{0}$ & $\mathbf{0}$ & 2 & 0 & $\mathbf{0}$ & 4 & 6 & 50 \\
\hline Sloanea guianensis (Aubl.) Benth. & ecológico & sementes & 1 & 2 & 0 & 0 & 2 & 0 & 0 & 5 & 30 & 42 \\
\hline Sorocea bonplandii (Baill.) Burger, Lanj. & ecológico & sementes & 1 & 2 & 1 & 2 & 2 & 2 & 1 & 11 & 39 & 01 \\
\hline et Boer & ornamental & sementes & 1 & 2 & 1 & 2 & 2 & 2 & 1 & 11 & 39 & 31 \\
\hline & alimentícia & frutos & 1 & 2 & 2 & 1 & 1 & 2 & 2 & 11 & & \\
\hline Svarrus romanzaffiang (Cham ) Glascman & apícola & flores & 1 & 2 & 2 & 1 & 2 & 2 & 2 & 12 & 26 & 42 \\
\hline 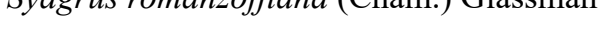 & artesanato & sementes & 1 & 2 & 2 & 1 & 2 & 2 & 2 & 12 & 20 & 42 \\
\hline & ecológico & sementes & 1 & 2 & 2 & 1 & 2 & 2 & 2 & 12 & & \\
\hline
\end{tabular}

Continua... 
TABELA 2: Continuação...

TABLE 2: Continued...

\begin{tabular}{|c|c|c|c|c|c|c|c|c|c|c|c|c|}
\hline Espécie & Usos & $\begin{array}{c}\text { Parte } \\
\text { utilizada }\end{array}$ & A & B & $\mathrm{C}$ & $\mathrm{D}$ & $\mathrm{E}$ & $\mathrm{F}$ & G & VPES & $\mathrm{Dm}$ & $\mathrm{FA}$ \\
\hline \multirow{5}{*}{ Syagrus romanzoffiana (Cham.) Glassman } & fibra & folhas & 2 & 2 & 2 & 1 & 2 & 2 & 2 & 13 & \multirow{5}{*}{26} & \multirow{5}{*}{42} \\
\hline & forrageira & folhas & 2 & 2 & 2 & 1 & 2 & 2 & 2 & 13 & & \\
\hline & medicinal & flores & 1 & 2 & 2 & 1 & 2 & 2 & 2 & 12 & & \\
\hline & \multirow{2}{*}{$\begin{array}{l}\text { ornamental } \\
\text { produto } \\
\text { bioquímico }\end{array}$} & sementes & 1 & 2 & 2 & 1 & 2 & 2 & 2 & 12 & & \\
\hline & & frutos & 1 & 2 & 2 & 1 & 1 & 2 & 2 & 11 & & \\
\hline Tetrorchidium rubrivenium Poepp. et Endl. & ecológico & sementes & 1 & 1 & 1 & 1 & 2 & 2 & 2 & 10 & 14 & 75 \\
\hline Trichilia lepidota Mart. & $\begin{array}{c}\text { produto } \\
\text { bioquímico }\end{array}$ & folhas & 2 & 1 & $\mathbf{0}$ & $\mathbf{0}$ & 0 & 0 & $\mathbf{0}$ & 3 & 15 & 67 \\
\hline \multirow{4}{*}{ Virola bicuhyba (Schott ex Spreng.) Warb. } & artesanato & sementes & 1 & 2 & 2 & 1 & 2 & 2 & 2 & 12 & \multirow{4}{*}{20} & \multirow{4}{*}{75} \\
\hline & medicinal & sementes & 1 & 2 & 2 & 1 & 1 & 2 & 2 & 11 & & \\
\hline & ornamental & sementes & 1 & 2 & 2 & 1 & 2 & 2 & 2 & 12 & & \\
\hline & $\begin{array}{l}\text { produto } \\
\text { bioquímico }\end{array}$ & cascas & 0 & 2 & 2 & 1 & 1 & 2 & 2 & 10 & & \\
\hline
\end{tabular}

citadas com maior número de vezes, entre elas Syagrus romanzoffiana, com nove citações, Euterpe edulis, Ilex paraguariensis e Psidium cattleianum, com sete, Cabralea canjerana, Cedrella fissilis e Luehea divaricata, com seis citações.

Os usos mais frequentes ocorrem para as categorias: ecológico (37\%), ornamental (24\%), medicinal $(10 \%)$, apícola $(8 \%)$ e produto bioquímico $(7 \%)$, seguidas por alimentícia $(6 \%)$, artesanato $(4 \%)$, forrageira $(3 \%)$ e fibra $(2 \%)$.

Dentre as partes vegetais utilizadas, as sementes aparecem com maior representatividade (65\%), fato esse devido à grande maioria das plantas se apresentarem como PFNM de origem ecológica e/ou ornamental. Isso deixa evidente o caráter produtor de mudas dessas plantas, já que elas podem ser utilizadas tanto para fins de recuperação de áreas degradadas como para fins ornamentais. $\mathrm{Na}$ sequência, a mais citada dentre os usos foram as folhas (13\%), principalmente pelo uso medicinal e de extração de produtos bioquímicos de tais plantas. Em seguida, apareceram as flores (9\%), que têm como principal razão para sua menção o fato de serem fundamentais no uso apícola, já que produzem pólen e néctar, atrativos de animais produtores de mel, como as abelhas; os frutos (7\%), que se destacaram como alimentícia, tanto na alimentação humana quanto animal, e a casca (6\%), largamente utilizada no uso medicinal.

Devido ao critério de densidade igual ou superior a 5 indivíduos.ha-1 ${ }^{-1}, 56$ espécies (59\%) obtiveram densidade média e 44 espécies (41\%) obtiveram densidade alta.

A produção de sementes e a taxa de crescimento foram critérios com menor número de informações disponíveis nas referências consultadas. Ficou evidente também a necessidade de ampliar o conhecimento ecológico das espécies arbóreas necessário para o seu manejo. No que diz respeito à taxa de produção de sementes, 32 espécies (40\%) possuem alta produção, 18 espécies (23\%) obtiveram moderada taxa de produção de sementes e nenhuma espécie obteve baixa ou irregular produção de sementes. No entanto, para 29 espécies (37\%) não existe conhecimento disponível relacionados à taxa de produção de sementes (ausência de informação). No que diz respeito à taxa de crescimento, para 40 espécies (49\%) não foi possível avaliar sua taxa de crescimento (ausência de informação), refletindo a falta de estudos ecofisiológicos das espécies pesquisadas.

Os conhecimentos ecológicos disponíveis mostraram que 11 espécies (14\%) obtiveram ausência de informação, 29 espécies (37\%) mostraram-se com pouca informação e 39 espécies (49\%) possuem alto conhecimento ecológico, incluindo informações sobre dinâmica populacional, biologia da reprodução e aspectos silviculturais.

Quanto à demanda de processamento, de 191 usos citados, 146 (77\%) representaram o consumo de produtos florestais não madeireiros in 
natura, ou seja, utilizam partes das plantas sem o emprego de beneficiamento que demande custo; 31 usos (16\%) foram citados com emprego de equipamento ou beneficiamento de baixo custo, e 14 usos (7\%) foram citados como sendo necessário à utilização de equipamentos de alto custo.

Das espécies analisadas, 40 espécies (51\%) não apresentaram níveis de injúria conhecidos ou não constaram informações sobre injúria, nos processos de coleta, manuseio ou consumo. No entanto, 38 espécies (48\%) apresentaram ausência de informação nas fontes pesquisadas, necessitando de estudos sobre possíveis efeitos tóxicos. Somente Sorocea bonplandii teve categorização de injúria mediana ou controlável, devido à apresentação de látex cáustico, que pode gerar toxicidade, se não controlado (CARVALHO, 2006). Se não for levada em consideração a ausência de informação, nenhum dos usos foi citado como causador de injúria severa ou não controlável, fato esse extremamente relevante, pois as espécies poderão ser utilizadas, sem ocorrer danos mais sérios, tanto aos seres humanos quanto aos animais.

Dentre os usos descritos para as espécies selecionadas, apenas Allophylus edulis apresentou o maior VPES, com 14, com indicação das folhas para uso medicinal.

Trinta e oito espécies atingiram VPES igual ou superior a 10 , sendo consideradas de elevado potencial de exploração sustentável de PFNM. Entre as espécies citadas como mais representativas destacou-se Syagrus romanzoffiana, popularmente conhecida como jerivá, amplamente distribuída pelos estados do sul e sudeste e com alta plasticidade ecológica, já que pode ser encontrada em três biomas brasileiros: Mata Atlântica, Cerrado e Pampa (CARVALHO, 2006). Entre os usos econômico e potencial apresenta-se como excelente planta ornamental, já que é a palmeira nativa mais utilizada na arborização de ruas e avenidas em todo o país, sendo de fácil transplante em qualquer idade, graças a seu sistema radicular ser superficial e bastante ramificado (CARVALHO, 2006).

Outra espécie expressiva foi Psidium cattleianum, conhecida popularmente por araçá, que possui fruto muito doce e suculento quando maduro (SANTOS; FERREIRA; ÁQUILA, 2004). Seus frutos representam grande potencial econômico, porém, ainda pouco explorado, já que não existe ainda oferta adequada à demanda deste fruto no mercado. No entanto, a industrialização, em forma de geleias e sorvetes, já se mostra interessante e com mercado promissor. $\mathrm{O}$ que ainda falta para o araçá é tornar-se um fruto de mesa, o que lhe daria maior visibilidade e aumentaria o seu consumo in natura (LISBOA; KINUPP; BARROS, 2011).

Euterpe edulis (palmiteiro) destacou-se por sua alta densidade nos estudos analisados. É uma das palmeiras mais representativas da Floresta Ombrófila Densa, por vezes dominante, com ampla distribuição na Mata Atlântica, e uma das espécies mais ameaçadas devido à exploração ilegal do palmito. Atualmente, alavancada pela utilização do fruto para obtenção do açaí em substituição ao açaíamazônico (Euterpe oleracea Mart.).

Com o hábito alimentar disseminado por todo o país, inclusive na região Sul, os agricultores passaram a comercializar frutos de Euterpe edulis para a produção do açaí (Açaí Mata Atlântica), aproveitando-se da semelhança com os frutos do norte (BOURSCHEID et al., 2011). Em Santa Catarina, os frutos de Euterpe edulis já vêm sendo coletados para a produção de açaí há mais de um século, desde 1870, quando muitas mulheres do norte do Estado difundiram o conhecimento (BOURSCHEID et al., 2011).

A produção de açaí não implica a morte da planta, como acontece no caso da extração do palmito, sendo relevante do ponto de vista dos produtos florestais não madeireiros, pois a produção pode ocorrer todos os anos e por longos períodos, diferentemente da extração do palmito, que ocorre uma única vez para cada palmeira. O cultivo do açaí poderia ser implementado pela agroindústria do sul de Santa Catarina, como já acontece no município de Garuva, onde existe o plantio desta palmeira para produção do açaí.

Em 2004, ano da criação da marca "Açaí Mata Atlântica", foi produzido em torno de 2.500 $\mathrm{kg}$ de polpa do fruto, aumentando sucessivamente sua produção todos os anos, tanto que em 2008 o aumento foi expressivo, passando para $48.000 \mathrm{~kg}$ de polpa de açaí. Além disso, Euterpe edulis é uma das espécies que bem representa o potencial de PFNM, pois gera lucro de maneira sustentável, garantindo a restauração florestal (BRANCALION et al., 2012).

Para indicação de uso ecológico das plantas, essas aqui elencadas, aparecem como de extrema utilidade para restauração de ambientes alterados. Muitas dessas espécies apresentaram atrativos para fauna, como no caso de Euterpe edulis e de Psidium cattleianum. Essas espécies apresentam frutos apreciados principalmente pelas aves. Assim que se alimentam, elas também dispersam as sementes, 
revelando, desta forma, seu caráter ecológico, além de outras espécies com a mesma característica (BACKES; IRGANG, 2002).

Quanto ao uso ornamental, as espécies citadas apresentaram características importantes para a arborização urbana, como por exemplo coloração das flores, frutos, tamanho e forma das copas, sistema radicular e ausência de substâncias tóxicas ou alérgicas (CAZNOK et al., 2010), como no caso de Cupania vernalis, ou até mesmo pela sombra que proporciona, como Inga sessilis (CAZNOK et al., 2010).

Sobre o uso medicinal das plantas, quando utilizadas de forma in natura, são usadas frequentemente no preparo de chás ou macerações, com intuito de extração do princípio ativo contido na casca ou nas folhas, na maioria das vezes, por cozimento, como citado para Casearia silvestris (SOUSA, 1991).

Algumas das espécies foram descritas como apícolas ou melíferas, pois se destacaram na produção de pólen e néctar, responsáveis pela fabricação de própolis e mel, conforme citado para Inga sessilis e Byrsonima ligustrifolia (CARVALHO, 2006).

Algumas espécies relacionadas com a alimentação humana mostraram-se de suma importância, com aproveitamento praticamente de todo o vegetal, já que se utilizam frutos, sementes, flores e até pétalas. Muitas vezes, tais plantas são utilizadas como condimento, nesse caso, tendo a extração a partir das sementes ou dos frutos. Em alguns casos, fica evidente a melhoria na qualidade do produto através do beneficiamento de tais alimentos pela indústria, o que facilita muito a sua comercialização. Além da alimentação humana também aparecem descritas espécies responsáveis pela nutrição de animais domésticos e na pecuária. As plantas denominadas forrageiras apresentam proteína bruta e baixa concentração de tanino (CORANDIN, 2011).

O uso de plantas para artesanato é citado em poucas espécies, dentre elas se destaca Syagrus romanzoffiana. $\mathrm{O}$ uso de tais vegetais aparece geralmente aliado às partes mais secas da planta, como no caso dos galhos e ramos mais finos. $\mathrm{O}$ uso de tais segmentos leva à produção de cestos, bijuterias, peças de adorno, esculturas, entre outros. Aliados quase sempre ao artesanato apareceram plantas reportadas como fibrosas, que são aquelas usadas na fabricação de roupas, redes, cordas, barbantes e utensílios afins, destacando-se também
Anonna sericea (CORANDIN, 2011).

Alguns outros usos foram reportados para algumas espécies, os quais não se adequavam a nenhuma das categorias anteriores, como Ilex paraguariensis, planta usada na fabricação de cosméticos (DICKEL; RITTER; BARROS, 2011).

Cabe salientar que outros dois trabalhos, com mesma metodologia utilizada no presente estudo, foram realizados no Estado do Paraná. O primeiro descreve a potencialidade de uso como PFNM de 70 espécies arbóreas em Floresta Ombrófila Mista (MARQUES, 2008) e o segundo descreve 58 espécies associadas à remanescente florestal ripário em Floresta Estacional Semidecidual (UBESSIMACARINI; NEGRELLE; SOUZA, 2011).

Constatou-se que o número de estudos florísticos e fitossociológicos realizados recentemente (últimos 17 anos) no Sul do Estado de Santa Catarina, em proporção ao número de municípios, é de pequena expressão, já que apenas oito municípios, dos 44 existentes, apresentaram algum estudo fitossociológico concluído. Este fato gera preocupação em virtude da degradação ambiental da região e consequente fragmentação florestal.

\section{CONCLUSÕES}

Os resultados obtidos evidenciam ser possível a conservação e desenvolvimento regional, a partir de alternativas sustentáveis de uso dos recursos florestais.

Dentre as 79 espécies avaliadas, todas possuem indicação de pelo menos um uso não madeireiro. Desse total, 38 atingiram valor potencial de exploração sustentável (VPES) igual ou superior a 10.

Destacam-se para uso como PFNM, com valor 14 de VPES, somente Allophylus edulis, seguida de Euterpe edulis, Garcinia gardineriana, Jacaranda puberula, Meliosma sellowii, Psidium cattleianum e Syagrus romanzoffiana, com valor 13 de VPES.

\section{REFERÊNCIAS BIBLIOGRÁFICAS}

AleXiades, M. N.; SHANLey, P. Productos forestales, medios de subsistencia y conservación. Bogor: Cifor, 2004.

BACKES, P.; IRGANG, B. E. Árvores do sul: guia de identificação e interesse ecológico. Santa Cruz do Sul: Ed. Clube da Árvore, 2002. 
BALZON, D. R.; SILVA, J. C. G. L.; SANTOS, A. J. Aspectos mercadológicos de produtos florestais não madeireiros: análise retrospectiva. Floresta, Curitiba, v. 34, n. 3, p. 363-71, 2004.

BOSA, D. M. Composição florística e estrutural de comunidade arbórea de Floresta Ombrófila Densa Montana no município de Morro Grande, Santa Catarina. 2011. 87 f. Dissertação (Mestrado em Ciências Ambientais) - Universidade do Extremo Sul Catarinense, Criciúma, 2011.

BOURSCHEID, K. et al. Euterpe edulis. In: CORADIN, L.; SIMINSKI, A.; REIS, A. (Ed.). Espécies nativas da flora brasileira de valor econômico atual ou potencial: plantas para o futuro - Região Sul. Brasília: MMA, 2011. p. 178-183.

BRANCALION, P. H. S. et al. Finding the Money for tropical Forest restoration. Unasylva 239, Rome, v. 63, p. 25-34, 2012.

CARVALHO, P. E. R. Espécies arbóreas brasileiras. Brasília: Embrapa Informação Tecnológica/Embrapa Florestas, 2006.

CAZNOK, J. et al. Arborização das cidades: direito à biodiversidade e à cidade saudável - uma proposta para Criciúma (SC). In: GONÇALVES, T. M.; SANTOS, R. Cidade e Meio Ambiente: estudos interdisciplinares. Criciúma: UNESC, 2010. p. 255-286.

CITADINI-ZANETTE, V. Florística, fitossociologia e aspectos da dinâmica de um remanescente de Mata Atlântica na microbacia do rio Novo, Orleans, SC. São Carlos, 249 f. Tese (Doutorado em Ecologia e Recursos Naturais) Universidade Federal de São Carlos, São Carlos, 1995.

COLONETTI, S. et al. Florística e estrutura fitossociológica em Floresta Ombrófila Densa Submontana na barragem do rio São Bento, Siderópolis, Estado de Santa Catarina. Biological Scienses, Acta Scientiarum, v. 31, n. 4, p. 397-405, 2009.

CORADIN, L.; SIMINSKI, A.; REIS, A. (Ed.). Espécies nativas da flora brasileira de valor econômico atual ou potencial: plantas para o futuro - Região Sul. Brasília: MMA, 2011.

DICKEL, M. L.; RITTER, M. R.; BARROS, I. B. I. Ilex paraguariensis. In: CORADIN, L.; SIMINSKI, A.; REIS, A. (Ed.). Espécies nativas da flora brasileira de valor econômico atual ou potencial: plantas para o futuro - Região Sul. Brasília: MMA, 2011. P. 632-635.

EMERICH, K. H. Composição florística e relação entre variáveis ambientais e a estrutura da comunidade arbórea de fragmento florestal ciliar do Rio Turvo, município de Turvo, Santa Catarina. 2009. 81 f. Dissertação (Mestrado em Ciências Ambientais) - Universidade do Extremo Sul Catarinense, Criciúma, 2009.

FAO. Food and Agriculture Organization of the United Nations. Consulta de expertos sobre productos forestales no madereros para América Latina y el Caribe. San Tiago: FAO, 1994.

FAO. Food and Agriculture Organization of the United Nations. Non-wood forest products for rural income and sustainable forestry. Rome: FAO, 1995.

FAO. Food and Agriculture Organization of United Nations. Products forestales no madereros: possibilidades futuras. Roma: Estudio FAO Montes 97, 1992.

FDA. Food and Drug Administration. FDA Poisonous Plant Database. Disponível em: $<w w w$. accessdata.fda.gov/scripts/platox/index.cfm>. Acesso em: 15 jul. 2011.

GRIMES, A.; LOOMIS, S.; JAHNIGE, P. Valuing the Rain Forest: the economic value of nontimber forest products in Ecuador. Ambio, v. 23, n. 7, p. 405-410, 1994.

IBGE. Instituto Brasileiro de Geografia e Estatística. Cidades. Disponível em: <www.ibge.gov.br/ cidadesat/painel/painel.php? codmun $=420460>$. Acesso em: 25 mar. 2013.

LISBOA G. N.; KINUPP, V. F.; BARROS, I. B. I. Psidium cattleianum. In: CORADIN, L.; SIMINSKI, A.; REIS, A. (Ed.). Espécies nativas da flora brasileira de valor econômico atual ou potencial: plantas para o futuro - Região Sul. Brasília: MMA, 2011. p. 205-208.

MARQUES, T. P. Recuperação florestal com o uso de não madeiráveis. Curitiba: Secretaria de Estado de Meio Ambiente e Recursos Hídricos do Estado do Paraná, Paraná Biodiversidade, 2008.

MARTINS, R. Composição e estrutura vegetacional em diferentes formações na floresta Atlântica, Sul de Santa Catarina, Brasil. 2010. $151 \mathrm{f}$. Tese (Doutorado) - Universidade Federal do Rio Grande do Sul, Porto Alegre, 2010.

MARTINS, R. Florística, estrutura fitossociológica e interações interespecíficas de um remanescente de Floresta Ombrófila Densa como subsídio para recuperação de áreas degradadas pela mineração de carvão, Siderópolis, SC. 2005.94 f. Dissertação (Mestrado em Biologia Vegetal) - Universidade Federal de Santa Catarina, Florianópolis, 2005. NEPSTAD, D. C.; SCHWARZMA, S. Non-timber 
products from tropical forests: evaluation of a conservation and development strategy. New York: New York Botanic Garden, 1992.

PACHECO, D. Planejamento para infraestrutura de trilhas em fragmento florestal urbano no município de Criciúma, Santa Catarina. 2010. 80

f. Dissertação (Mestrado em Ciências Ambientais) - Universidade do Extremo Sul Catarinense, Criciúma, 2010.

PASETTO, M. R. Composição florística e estrutura de fragmento de floresta ombrófila densa submontana no município de Siderópolis, Santa Catarina. 2008. 44 f. Trabalho de Conclusão de Curso (Ciências Biológicas) - Universidade do Extremo Sul Catarinense, Criciúma, 2008.

REBELO, M. A. Florística e fitossociologia de um remanescente florestal ciliar: subsídio para a reabilitação de vegetação ciliar para microbacia do rio Três Cachoeiras, Laguna, SC. 2006. 143 f. Dissertação (Mestrado em Ciências Ambientais) - Universidade do Extremo Sul Catarinense, Criciúma, 2006.

SANTOS, A. J. et al. Produtos não madeireiros: comercialização, classificação, valoração e mercados. Revista Floresta, Curitiba, v. 33, n. 2, p. 215-224, 2003.

SANTOS, C. M. R.; FERREIRA, A. G.; ÁQUILA, E. A.. Características de frutos e germinação de seis espécies de Myrtaceae nativas do Rio Grande do Sul. Ciência Florestal, Santa Maria, v. 14, n. 2 , p.13-20, 2004.

SANTOS, R. Reabilitação de ecossistemas degradados pela mineração de carvão a céu aberto em Santa Catarina, Brasil. 115 f. Tese (Doutorado em Engenharia Mineral). Escola
Politécnica da Universidade de São Paulo, São Paulo, 2003.

SILVA, R. T. Florística e estrutura da sinúsia arbórea de um fragmento urbano de Floresta Ombrófila Densa do município de Criciúma, Santa Catarina. 2006. 61 f. Dissertação (Mestrado em Ciências Ambientais) - Universidade do Extremo Sul Catarinense, Criciúma, 2006.

SIMÕES, C. O. et al. Plantas da medicina popular do Rio Grande do Sul. 5. ed. Porto Alegre: Editora da UFRGS, 1998.

SOUSA, M. P. et al. Constituintes químicos ativos de plantas medicinais brasileiras. Fortaleza: EUFC, 1991.

STATZ, J. Non-timber forest products: a key to sustainable tropical forest management? Gate Technology and Development, n. 2, p. 4-11, 1997. UBESSI-MACARINI, C.; NEGRELLE, R. R. B.; SOUZA, M. C. Produtos florestais não-madeiráveis e respectivo potencial de exploração sustentável, associados à remanescente florestal ripário do alto rio Paraná, Brasil. Acta Scientiarum, Biological Sciences, Maringá, v. 33, n. 4, p. 451-462, 2011.

VANTOMME, P. Production and trade opportunities for non-wood forest products, particularly food products for niche markets. Geneva: FAO, 2001. Disponível em: http://www. fao.org/forestry/FOP/FOPW/NWFP/nwfp-e.stm. Acesso em: 25 mar. 2013.

VILLALOBOS, R.; OCAMPO, R. Productos no maderables del bosque en Centroamérica y el Caribe. Costa Rica: CATIE/OLAFO, 1997.

WONG, J. L. G.; THORNBER, K.; BAKER, N. Resource assessment of non-wood forest products. Rome: FAO, 2001. 